\title{
Cell-Engineered Nanovesicle as a Surrogate Inducer of Contact-Dependent Stimuli
}

\author{
Junho Kim, Chugmin Han, Wonju Jo, Sehong Kang, Siwoo Cho, Dayeong Jeong, \\ Yong Song Gho, and Jaesung Park**
}

Heterotypic interactions between cells are crucial in various biological phenomena. Particularly, stimuli that regulate embryonic stem cell (ESC) fate are often provided from neighboring cells. However, except for feeder cultures, no practical methods are identified that can provide ESCs with contact-dependent cell stimuli. To induce contact-dependent cell stimuli in the absence of living cells, a novel method that utilizes cell-engineered nanovesicles (CNVs) that are made by extruding living cells through microporous membranes is described. Protein compositions of CNVs are similar to their originating cells, as well as freely diffusible and precisely scalable. Treatment of CNVs produced from three different stromal cells successfully induces the same effect as feeder cultures. The results suggest that the effects of CNVs are mainly mediated by membrane-associated components. The use of CNVs might constitute a novel and efficient tool for ESC research.

\section{Introduction}

Heterotypic interactions between cells play crucial roles in the developmental and immunological processes of multicellular organisms. ${ }^{[1-3]}$ For example, stimuli for lineage-specific differentiation of embryonic stem cells (ESCs) are often provided by heterotypic interactions. ${ }^{[3,4]}$ Cells in a shared space continuously exchange stimuli through direct cell-cell contacts (juxtacrine stimuli) and/or secreting soluble factors (paracrine stimuli). ${ }^{[5,6]}$ Therefore, recreating these interactions in a laboratory setting is essential in many research fields, as well as cell cultures. The most commonly used method to reconstitute

Dr. J. Kim, D. Jeong, Prof. J. Park

School of Interdisciplinary Bioscience and Bioengineering POSTECH

77 Cheongam-Ro Nam-gu, Engineering Building 5, Pohang

Gyeong-buk 37673, Republic of Korea

E-mail:.jpark@postech.ac.kr

C. Han, W. Jo, S. Kang, S. Cho, Prof. J. Park

Department of Mechanical Engineering

POSTECH

77 Cheongam-Ro Nam-gu, Engineering Building 5, Pohang

Gyeong-buk 37673, Republic of Korea

Prof. Y. S. Gho

Department of Life Sciences

POSTECH

77 Cheongam-Ro Nam-gu, BIOTECH CENTER, Pohang

Gyeong-buk 37673, Republic of Korea

DOI: 10.1002/adhm.201700381 cell-cell interactions in an in vitro condition is cocultures. ${ }^{[4,7]}$ However, different cell types inevitably mix in cocultures, and this contamination often complicates analyses and obscures results. ${ }^{[8,9]}$ In addition, because of the complex and uncontrollable nature of the method, it is difficult to precisely manipulate the degree of heterotypic interactions.

ESCs are often regulated by stimuli from feeder cultures. For example, stimuli from mouse embryonic fibroblasts ( $\mathrm{mEFs}$ ) contribute to the self-renewal of ESCs, and stimuli from OP9 and PA6 cells contribute to hematopoietic and neuronal lineagespecific differentiation of ESCs, respectively. ${ }^{[10-12]}$ Although soluble factors, such as cytokines, and small chemicals, such as retinoic acids, have been purified and used for feeder-free cultures of ESCs, no other methods currently exist that provide cells with contact-dependent stimuli, except the coculture method. ${ }^{[13-15]}$ Therefore, a continuing need exists for a new method to overcome the problems of the coculture method in ESC research.

A number of methods have been developed to meet this need in ESC research. Some studies showed that a part of contactdependent stimuli can be replaced by different combinations of extracellular matrices (ECMs). ${ }^{[16-18]}$ However, ECMs cannot provide cell-specific stimuli which are mediated by membraneassociated proteins, and these approaches have been utilized only in limited numbers of applications. ${ }^{[19]}$ Some recent studies have also utilized chemically fixed feeder layers, but they still did not successfully recreate cell-specific contact-dependent stimuli and may cause undesired effects by fixatives. ${ }^{[20,21]}$

As a viable alternative, we developed a method that uses cell-engineered nanovesicles (CNVs), which possess similar lipid and protein compositions to their originating cells. To produce such vesicles, we applied the serial extrusion method to cells and separated vesicle fractions by density-gradient ultracentrifugation, which has been previously used to produce liposomes. ${ }^{[22,23]}$ Produced CNVs are freely diffusible in culture media, and can be precisely quantified in terms of particle counts or protein amounts. By treating ESCs with CNVs made from mEF, OP9 and PA6 stromal cells, we can induce feeder-related ESC regulations in the absence of living cells. In addition, from the result that $\mathrm{CNV}^{\mathrm{mEF}}$ was fully functional only in the presence of leukemia inhibitory factor (LIF), we confirmed that the effects of CNV treatment are mainly induced 
by the physical contacts, and that soluble factors are of lesser importance.

\section{Results}

\subsection{Characterization of CNVs}

Three different stromal cells (mEF, OP9, and PA6) were serially extruded through track-etched polycarbonate filters having 10 and $5 \mu \mathrm{m}$ pores. The extruded solutions containing vesicles were then subjected to Opti-prep density-gradient ultracentrifugation. CNV fractions were separated from the interface between $10 \%$ and $30 \%$ density solutions (Figure 1a). Purified CNVs were then quantified using Bradford protein assay, and aliquots of CNVs were stored at $-80{ }^{\circ} \mathrm{C}$ for up to three months for further use. The stability of stored CNVs was confirmed by comparing the size and protein profiles of fresh and stored CNVs (Figure S1, Supporting Information). Each batch of CNVs was confirmed not to have any viable cells prior to storage to prevent unwanted effects caused by cell-mixings (Figure S2, Supporting Information).

The morphologies of CNVs made from $\mathrm{mEF}\left(\mathrm{CNV}^{\mathrm{mEF}}\right)$, OP9 $\left(\mathrm{CNV}^{\mathrm{OP9}}\right)$, and PA6 $\left(\mathrm{CNV}^{\mathrm{PA}}\right)$ were confirmed using transmission electron microscopy (TEM) with uranyl acetate negative staining. All three types had similar diameters of 100-200 nm, and were generally spherical structures enclosed by lipid membranes (Figure 1b). Nanoparticle tracking analysis (NTA) results of the three CNVs were consistent with TEM results; their sizes ranged from $\approx 50 \mathrm{~nm}$ to a few hundred nanometers, with a peak at $\approx 150 \mathrm{~nm}$ (Figure $1 \mathrm{c}$ ). Coomassie blue staining of sodium dodecyl sulfate polyacrylamide gel (SDS-PAGE) results of cell and CNV lysates showed almost no differences; this similarity means that the protein compositions of CNVs were the same as those of their originating cells (Figure 1f). However, it also revealed that the protein composition of extracellular vesicles (EVs) was different from those of both the cells and CNVs (Figure 1f). When CNVs were produced from a cellline that stably expresses palmitoylated-mCherry (localized at the plasma membrane) and GFP (localized in the cytoplasm),

a)

Production of CNVs

Purification of CNVs

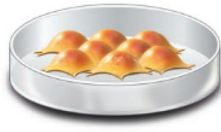

Feeder cells

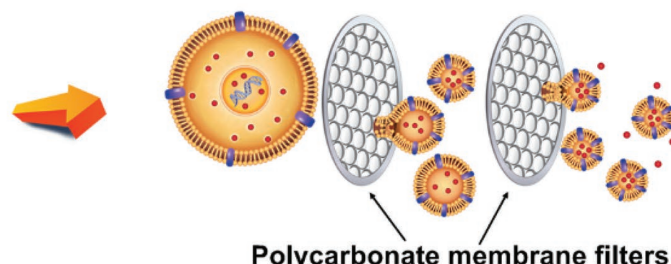

Polycarbonate membrane filters

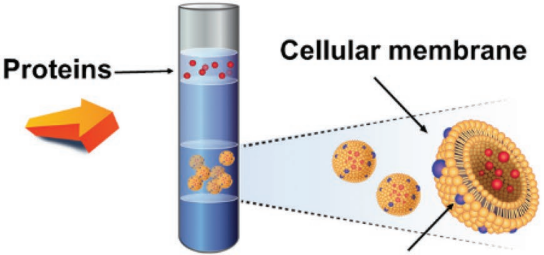

Membrane protein b)
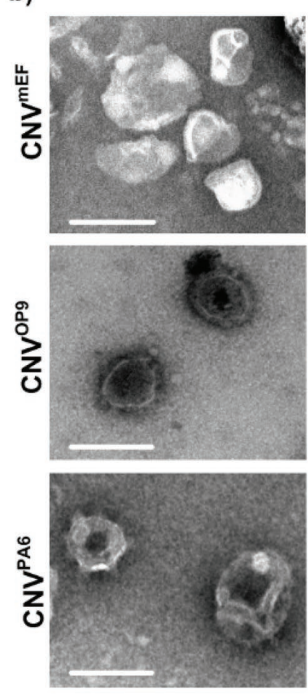

c)

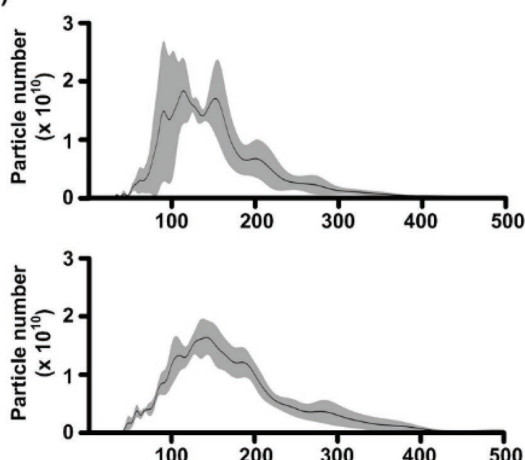

d)

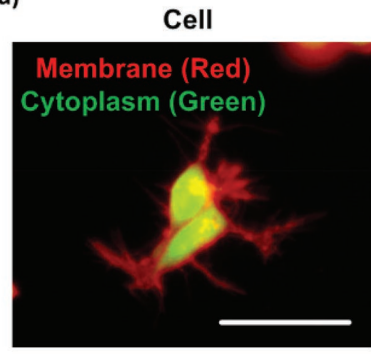

CNV

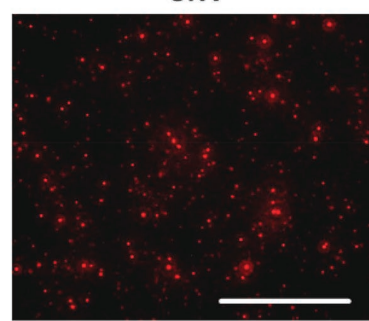

e)

f)

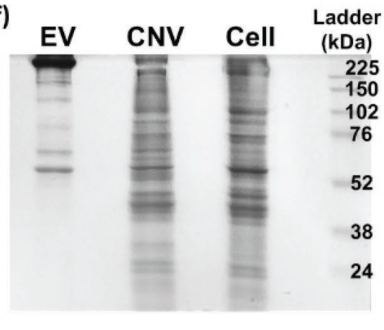

Figure 1. Production and characterization of CNVs. a) Schematic of CNV production. Cells were collected from cultures and serially extruded through track-etched microporous filters. CNVs were isolated from the extrudate by using density gradient ultracentrifugation. b) Transmission electron microscopic visualization of CNVmEF, CNVOP9, and CNVPA6. Scale bars: $200 \mathrm{~nm}$. c) Nanoparticle tracking analysis. The sizes of produced CNVs (CNVmEF, $\mathrm{CNV}^{\mathrm{OP} 9}$, and $\mathrm{CNV}^{\mathrm{PA} 6}$ ) were measured using NTA. Black solid lines: mean values; gray areas: \pm S.D. d) Membrane-to-cytoplasm ratio of CNVs. HEK293 cells that stably expressed cytoplasmic GFP and palmitoylated mCherry, and CNVs made from this HEK293 cells were imaged under epifluorescence microscope (images). Scale bars: $50 \mu \mathrm{m}$; Error bars: +S.D. e) The membrane-to-cytoplasm (mCherry-to-GFP) ratio was quantified using a multi-plate reader (graph). f) Coomassie blue analysis of EVs, CNVs, and cell. The SDS-PAGE patterns of CNVs and cells were very similar, but the SDS-PAGE pattern of EVs was completely different from them. 
a)
Cell - Cell Interaction

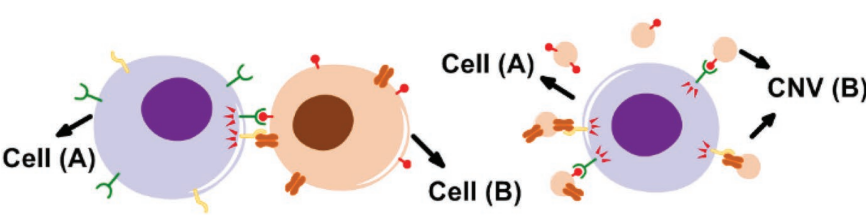

b)

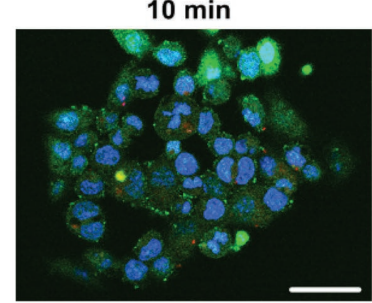

d)

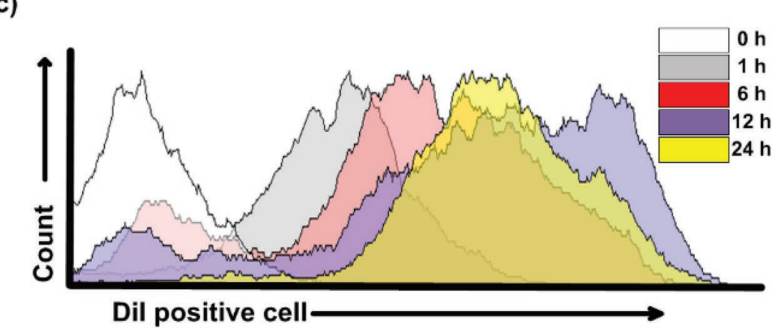

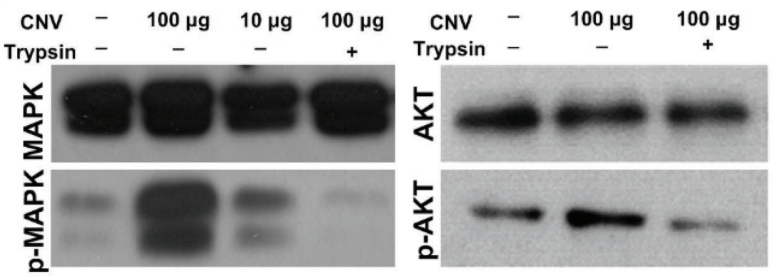

Figure 2. Characterization of CNVs and of cell-CNV interaction. a) Schematic of CNV-cell interaction. When cells and CNVs are closely located, they physically interact. b) Confocal microscopic analysis of CNV-cell interaction. CFSE (green) labeled ESCs were treated with Dil (red) labeled CNVmEF. Dil-labeled CNVs almost completely surrounded the cells $30 \mathrm{~min}$ after treatment. Scale bars: $50 \mu \mathrm{m}$. c) Flow cytometric analysis of CNV-cell interaction. More than half of the cells were positive for Dil-labeled CNV signals at $1 \mathrm{~h}$ after the treatment, and most cells were positive for the signal at $12 \mathrm{~h}$ after treatment. d) Phosphorylation of Mitogen- Activated Protein Kinase (MAPK) by short-term CNV treatment (left). mEFs were treated with ES CNVs for $30 \mathrm{~min}$. The phosphorylation of MAPK increased in a dose-dependent manner. Treatment with trypsinized CNVs did not induce phosphorylation of MAPK. Phosphorylation of Protein Kinase B (AKT) by long-term CNV treatment (right). ESCs were treated with CNVmEF five times for $10 \mathrm{~d}$. The phosphorylation of AKT was increased by these treatments. Treatment using trypsinized CNVs did not induce phosphorylation of AKT.

CNVs exhibited an $\approx 10$ times higher membrane-to-cytoplasm (mCherry-to-GFP) ratio than did their originating cells (Figure 1d,e).

\subsection{Characterization of Cell-CNV Interaction}

Produced CNVs were mixed with cell-culture media and applied to in vitro cultured cells. CNVs located near cells can come into physical contact with them. If the surface proteins of CNVs and the cells interact, the CNVs and cells will bind and exchange stimuli (Figure 2a). To identify the presence of such interaction between cells and CNVs, we treated cells with DiI-labeled CNVs. First, we used confocal microscopy to visually confirm the physical contacts between the cells and CNVs. 10 min after CNV treatment, red fluorescent signals of DiI-stained CNVs emerged, and 30 min after CNV treatment, most of the cells made physical contacts with CNV with red fluorescence signals (Figure 2b). To quantify the degree of cell-CNV interaction, we utilized flow cytometry to analyze the DiI-CNV-treated cells. The DiI signal accumulated until $12 \mathrm{~h}$ after treatment, and then plateaued and decreased slightly (Figure $2 \mathrm{c}$ ).

To determine whether the cell-CNV interactions can induce downstream signal transduction pathways, we performed western phosphorylation activity in short-term culture with a CNV treatment, and for AKT phosphorylation activity in longterm culture with repeated CNV treatments. In western blots after a CNV treatment, the amount of p-MAPK noticeably was increased in the treated cells, and in western blots after multiple CNV treatments, the amount of p-AKT was increased slightly in the treated cells (Figure 2d). In cells treated with CNVs made from cells that had been treated with trypsin for $1 \mathrm{~h}$, the phosphorylation of both the MAPK and AKT was significantly reduced; this result implies that phosphorylation of MAPK and AKT was induced by proteins on the CNV surfaces (Figure 2d).

\subsection{CNV ${ }^{m E F}$ Treatment for ESC Self-Renewal}

CNVs were produced from in vitro cultured mEFs. To test the ability of $\mathrm{CNV}^{\mathrm{mEF}}$ to maintain pluripotency, we examined the morphology and OCT3/4 expressions of ESCs treated with $\mathrm{CNV}^{\mathrm{mEF}}$. First, we performed the experiment in the absence of LIF, which is a crucial soluble factor that helps ESC to maintain their pluripotency. ${ }^{[14]}$ On the 3rd day, the CNV-treated groups showed no increase in OCT3/4 expression, which was significantly lower than that of the ESCs cultured with mEF feeder layers (Figure $3 \mathrm{a}$ ). In both the $\mathrm{CNV}^{\mathrm{mEF}}$-treated groups and nontreated groups, the cell morphologies dramatically changed, and their doubling times increased noticeably; these results imply that the ESCs had lost their self-renewing abilities and had begun to differentiate (data not shown).

We performed a similar experiment in the presence of LIF. In this condition, $\mathrm{CNV}^{\mathrm{mEF}}$ successfully maintained the pluripotency of ESCs. Under a phase-contrast microscope, the groups treated with $\geq 50 \mu \mathrm{g} \mathrm{mL}^{-1} \mathrm{CNV}^{\mathrm{mEF}}$ exhibited well-maintained dome-like ESC morphologies until the 12th day of culture (Figure S4a, Supporting Information). Flow cytometric analyses of OCT3/4 protein expression of the same cells showed similar results; groups treated with 50 and $100 \mu \mathrm{g} \mathrm{mL}^{-1} \mathrm{CNV}^{\mathrm{mEF}}$ showed similar or even higher OCT3/4 protein expressions than did feeder-culture groups (Figure 3b). Treatment of CNV also reduced the doubling time of ESC to a level similar to that of ESCs cultured on a mEF feeder layer (Figure S5, Supporting Information). The number of cells in the nontreated groups 
a)

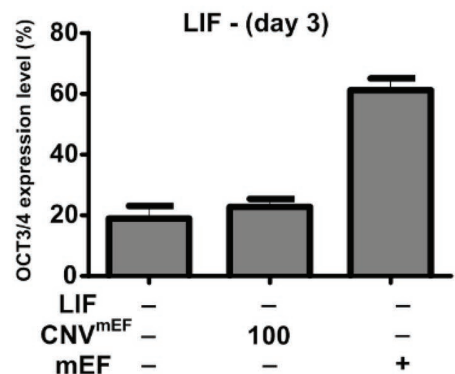

b)

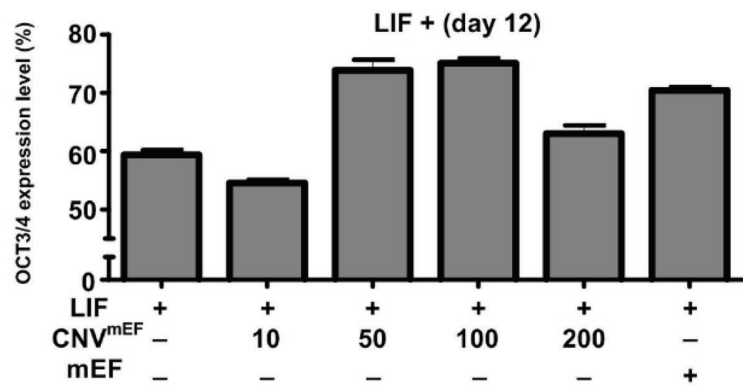

c)

ALP (day 24)
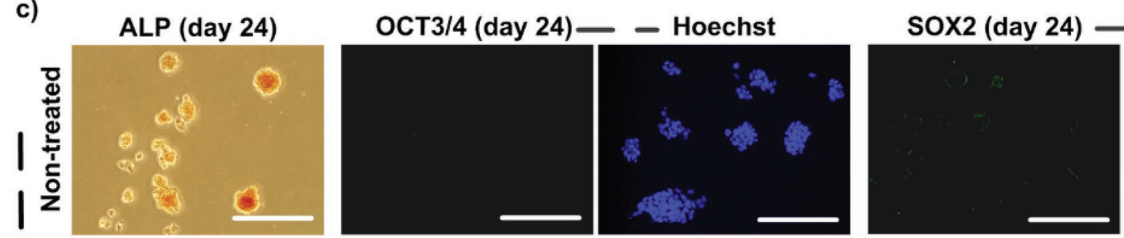

- Hoechst
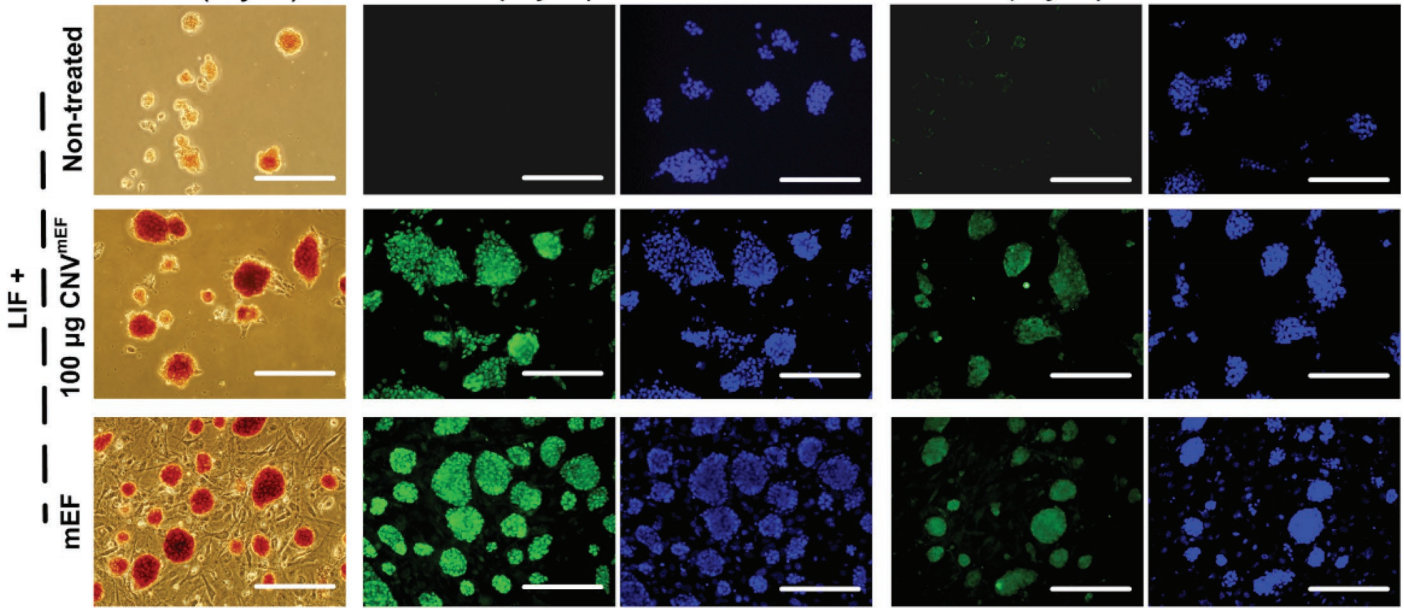

d)

Oct $3 / 4$

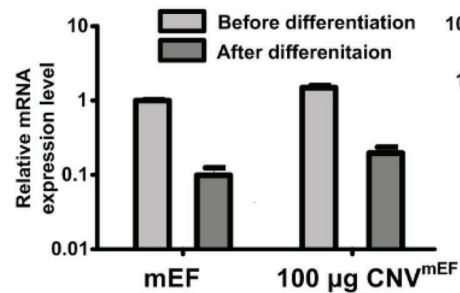

Hand 1

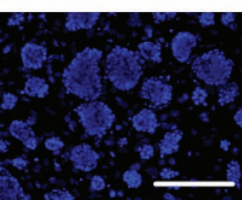

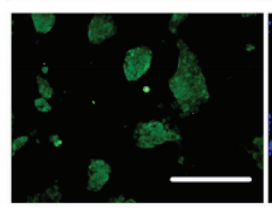

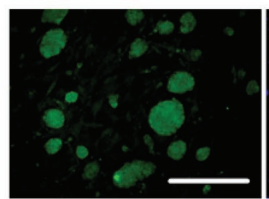

Sox1

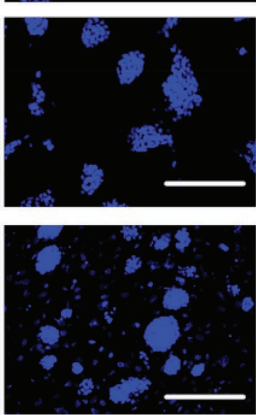

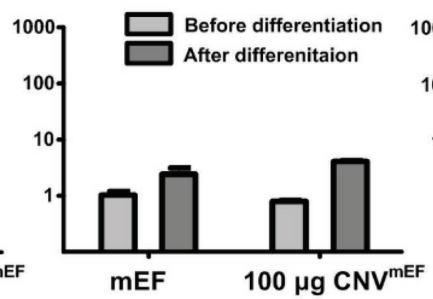
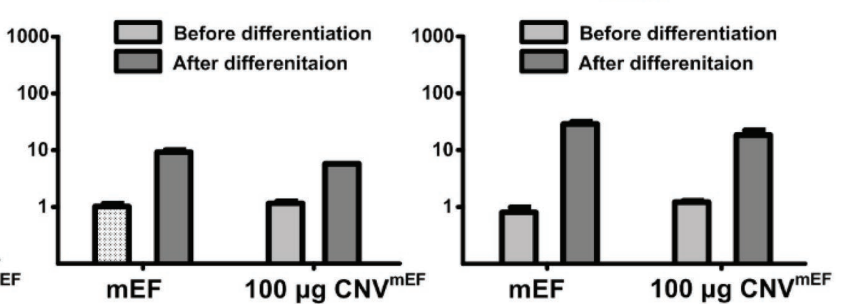

Figure 3. Pluripotency maintenance of ESCs after treatment with CNVmEF. a) Flow cytometric analysis of OCT3/4 transcription factors after CNVmEF treatment in the absence of LIF. After two CNVmEF treatments over $3 \mathrm{~d}$, the percentage of ESCs that expressed pluripotency-related protein OCT3/4 was

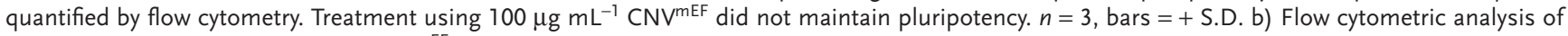
OCT3/4 transcription factors after CNVmEF treatment in the presence of LIF. After six CNV treatments for $12 \mathrm{~d}$, the percentage of ESCs that expressed OCT3/4 was measured using flow cytometry. Treatment using $100 \mu \mathrm{g} \mathrm{mL}^{-1} \mathrm{CNV}^{\mathrm{mEF}}$ was found to be optimal for maintenance of ESC pluripotency. $n=3$, bars $=+$ S.D. c) Microscopic analysis of pluripotency markers. ALP staining and immunofluorescent labeling of OCT3/4 and SOX2 were performed using ESCs cultured for $24 \mathrm{~d}$ in different conditions. The expressions of ALP, OCT3/4, and SOX2 in ESCs treated with $100 \mu \mathrm{g} \mathrm{mL} \mathrm{L}^{-1} \mathrm{CNV}^{\mathrm{mEF}}$ were similar to those of appropriately maintained ESCs. The nontreated group showed decreased ALP expression, and OCT3/4 and SOX2 expressions were absent. Scale bars: $200 \mu \mathrm{m}$. d) Relative mRNA expression level of Oct3/4, Hand1, Sox1, and Gata4 in differentiated ESCs that had been maintained with $\mathrm{CNV} \mathrm{mEF}^{\mathrm{m}}$ and $\mathrm{mEF}$ feeders. ESCs maintained with $\mathrm{CNV}^{\mathrm{mEF}}$ showed almost an identical degree of three germ layer differentiation abilities as those of appropriately maintained ESCs. $n=3$, error bars: +S.D.

decreased substantially because they had differentiated and lost their self-renewing ability.

To further confirm the efficiency of $\mathrm{CNV}^{\mathrm{mEF}}$ during a prolonged culture, we performed alkaline phosphatase (ALP) staining and immunofluorescence labeling of OCT3/4 and SOX2 after $24 \mathrm{~d}$ of culture. In the mEF feeder culture groups and $\mathrm{CNV}^{\mathrm{mEF}}$-treated groups, the expressions of ALP, OCT3/4, and SOX2 were well maintained even after $24 \mathrm{~d}$ of culture. In the nontreated groups, however, ALP expression decreased, and
OCT3/4 and SOX2 expressions almost disappeared (Figure 3c). To evaluate the pluripotency of the cells, ESCs maintained using CNVs for $>21 \mathrm{~d}$ were exposed to a differentiation condition. The differentiation ability of CNV-treated ESCs was the same as that of properly-maintained ESCs. They successfully differentiated into all three germ layers, and the mRNA expression levels of early germ-layer markers were almost identical to those of properly maintained ESCs (Figure 3d; Figure S6, Supporting Information). ESCs maintained without $\mathrm{mEF}$ 
feeder layers or $\mathrm{CNV}^{\mathrm{mEF}}$ did not survive for $21 \mathrm{~d}$; they spontaneously differentiated during the culture period and failed to form embryoid bodies (data not shown).

\subsection{CNV ${ }^{\text {OP9 }}$ Treatment for the Hematopoietic Differentiation of ESC}

CNVs were produced from in vitro cultured OP9 cells, which are known to induce ESCs to differentiate into hematopoietic lineages. ${ }^{[11]}$ On the 1 st day of $\mathrm{CNV}$ treatment, culture media were changed to hematopoietic differentiation media. To optimize the CNV treatment condition, ESCs were treated with different amounts of $\mathrm{CNV}^{\mathrm{OP} 9}$ for $6 \mathrm{~d}$ of culture. On the 6th day, the groups that had been treated with $\geq 50 \mu \mathrm{g} \mathrm{mL}$ $\mathrm{CNV}^{\mathrm{OP} 9}$ exhibited distinct colonies of spherical and protruding cells, which also appeared in the OP9 feeder culture group (Figure S4b, Supporting Information). However, the highest dose $\left(200 \mu \mathrm{g} \mathrm{mL}^{-1}\right)$ seemed to be toxic to the ESCs (Figure S4b, Supporting Information). The cells were collected from the culture, and early mesodermal gene expressions of the cells were confirmed by Quantitative reverse transcription-Polymerase Chain Reaction (RT-qPCR). The expressions of Flk1 and Scl genes were increased in a dose-dependent manner by $\mathrm{CNV}^{\mathrm{OP} 9}$, but decreased at the dose of $200 \mu \mathrm{g} \mathrm{mL} \mathrm{m}^{-1}$. Therefore, the $100 \mu \mathrm{g} \mathrm{mL}^{-1} \mathrm{CNV}^{\mathrm{OP} 9}$ dose was found to be optimal for mesodermal lineage differentiation (Figure 4a).

On the 17th day of culture with $100 \mu \mathrm{g} \mathrm{mL}^{-1} \mathrm{CNV}^{\mathrm{OP} 9}$, the number of spherical and protruding hematopoietic lineage cells in the CNV-treated groups seemed to exceed the number of such cells in OP9 feeder cultures, but nontreated groups showed irregular morphologies and mixed types of cells a)

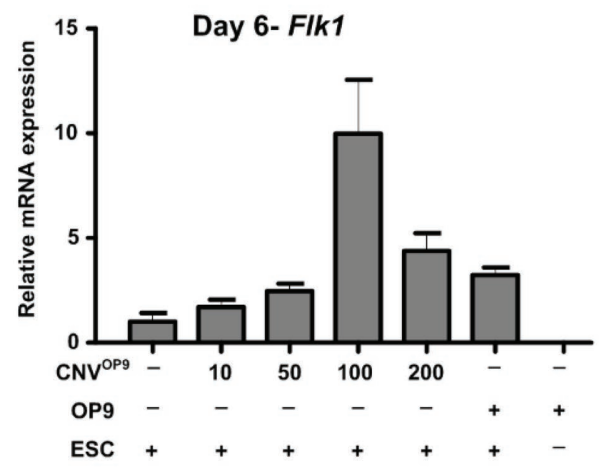

c)
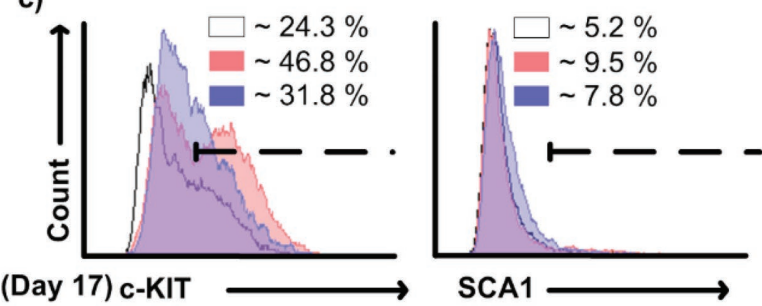

Day 6- Scl

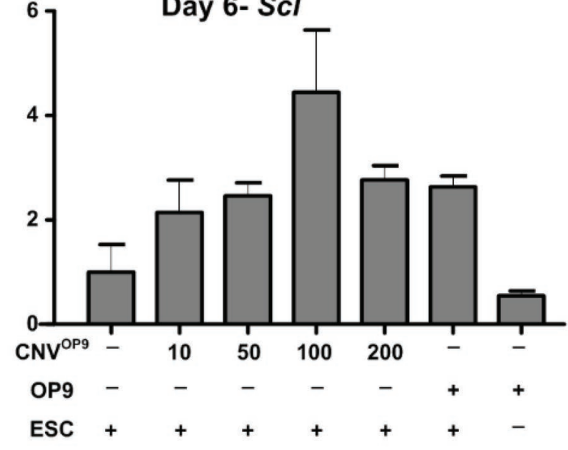

b)

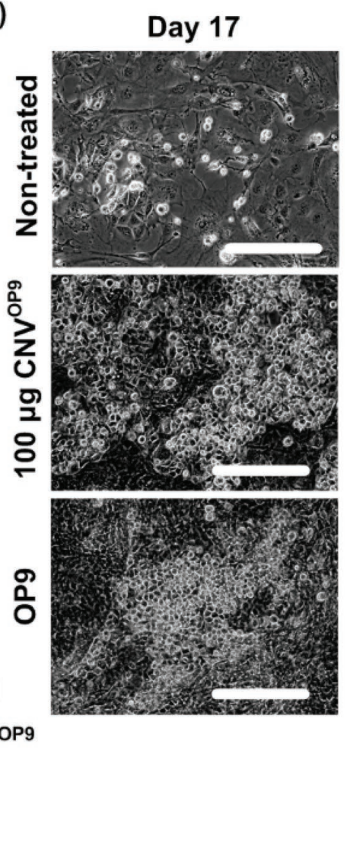

d)
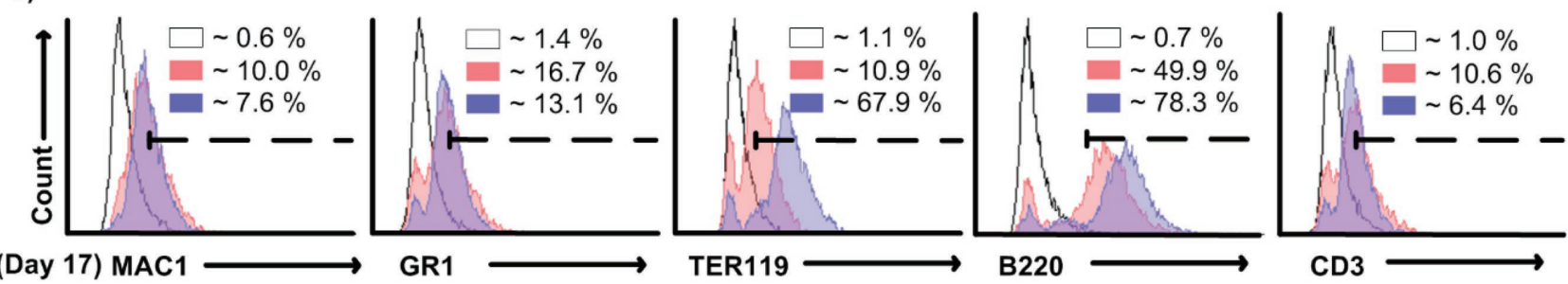

Figure 4. Hematopoietic differentiation of ESCs by treatment with CNVOP9. a) RT-qPCR analysis of mesodermal genes after CNVOP9 treatment. After two CNVOPg treatments, relative gene expressions of two mesodermal genes Flk1 and Scl were measured using RT-qPCR. Treatment using $100 \mu \mathrm{g} \mathrm{mL} \mathrm{C}^{-1} \mathrm{CNV}^{\mathrm{OP} 9}$ was optimal for mesodermal differentiation. $n=3$, bars: +S.D. b) Morphologies of ESCs cultured for $17 \mathrm{~d}$ with/without CNVOP9 . ESCs cultured without CNVOP9 showed heterogeneous morphologies of three germ layer cells on the 17th day of culture. ESCs cultured with CNVOP9 or OP9 feeder layers showed homogeneous blood cell-like morphologies on the 17th day of the culture. Scale bars: $200 \mu \mathrm{m}$. c) Flow-cytometry analyses of hematopoietic progenitor cell markers on the 17th day of the culture. Cells from the 17th day of the culture were stained with c-KIT, SCA1, and CD45 hematopoietic progenitor markers, and analyzed by flow cytometry. d) Flow cytometry analyses of mature blood cell markers on the 17th day of the culture. The same cells were also stained with MACl macrophage marker, GR1 granulocyte marker, TER119 erythrocyte marker, B220 B lymphocyte marker, and CD3 T lymphocyte marker, and then analyzed using a flow cytometer. 
(Figure 4b). Among the cells collected on the same day, hematopoietic progenitor cells that expressed c-KIT, SCA-1, and CD45 were detected by flow cytometric analyses. Among all of the groups, the CNV-treated groups exhibited the largest number of such cells (Figure 4c). On the 17th day, myeloid lineage cell (positive for Mac-1 macrophage marker, GR-1 granulocyte marker, and Ter119 erythrocyte marker) and lymphoid lineage cell (positive for B220 B lymphocyte marker and CD3 T lymphocyte marker) were also detected. The portions of these cells from the $\mathrm{CNV}^{\mathrm{OP} 9}$-treated groups were generally similar to the portion of the cells from feeder layer groups, but the nontreated cultures showed almost no $(<2 \%)$ mature hematopoietic lineage cells (Figure 4d). The existence of various hematopoietic lineage cells in the $\mathrm{CNV}^{\mathrm{OP} 9}$-treated and feeder layer groups was reconfirmed by DIFF-QUIK stained microscopic images (Figure S7, Supporting Information). Unlike the previous CNV ${ }^{\mathrm{mEF}}$ case, groups treated with $\mathrm{CNV}^{\mathrm{OP} 9}$ without the addition of additives, such as cytokines, exhibited similar or superior hematopoietic differentiation induction ability to that of the feeder groups.

\subsection{CNV ${ }^{O P 9}$ Treatment for the Neuronal Differentiation of ESC}

When PA6 cells are used as feeder layers, they induce ESCs to differentiate into neuronal cells. ${ }^{[12]}$ To test the ability of $\mathrm{CNV}^{\mathrm{PA} 6}$ to induce neuronal differentiation, we produced CNVs from in vitro cultured PA6 cells and applied them to ESCs with a neural differentiation medium. To determine the optimal condition for $\mathrm{CNV}^{\mathrm{PA} 6}$ treatment, ESCs were treated with different doses of $\mathrm{CNV}^{\mathrm{PA} 6}$ for $4 \mathrm{~d}$. Groups treated with $\mathrm{CNV}^{\mathrm{PA6}}$ showed comparatively mild phenotypic changes; as the dose of CNVs increased, the number of aggregated bodies increased, but cells treated with $200 \mu \mathrm{g} \mathrm{mL} \mathrm{m}^{-1} \mathrm{CNVs}$ seemed to be dying (Figure S4c, Supporting Information). The expression of ectodermal genes Nestin, Pax6, and Sox1 were analyzed in cells treated with $\mathrm{CNV}^{\mathrm{PA}}$. The expressions of all three genes increased in a manner dependent on CNV dose, except at $200 \mu \mathrm{g} \mathrm{mL}^{-1} \mathrm{CNV}^{\mathrm{PA}}$, in which case they decreased (Figure 5a). Because $100 \mu \mathrm{g} \mathrm{mL} \mathrm{m}^{-1} \mathrm{CNV}$-treated groups exhibited similar or higher ectodermal gene expressions to that of the feeder layer groups, the $100 \mu \mathrm{g} \mathrm{mL} \mathrm{m}^{-1}$ dose was optimal (Figure 5a).

On the 8th day of culture with the $100 \mu \mathrm{g} \mathrm{mL}^{-1} \mathrm{CNV}^{\mathrm{PA} 6}$ dose, ESCs showed comparatively homogenous spindle-like morphology; whereas, nontreated groups showed heterogeneous morphologies composed of all three germ-layer-like cells (Figure 5b). When these cells were labeled with neuron-specific TUJ1 antibodies, groups treated with $\mathrm{CNV}^{\mathrm{PA} 6}$ showed almost the same numbers and morphologies of neurons as groups treated with PA6 feeder layers, but nontreated groups showed far fewer TUJ1-positive cells than did the other two groups (Figure 5b). To quantitatively assess the efficacy of treatment with $\mathrm{CNV}^{\mathrm{PA}}$, we calculated the percentage of TUJ1-positive colonies in low-magnification fluorescent microscopic images. The percentage of TUJ1-positive colonies was almost the same in CNV-treated groups and feeder-culture groups, and both were significantly (one-way ANOVA; $F(2,6)=31.86, p<0.001$ ) $(\approx 5$ times) higher than in nontreated groups (Figure $5 \mathrm{c}$ ). Groups treated with $\mathrm{CNV}^{\mathrm{PA} 6}$ without any additional substances exhibited almost identical neural differentiation induction ability to that of the feeder cultures.

\section{Discussion}

CNVs were developed and used as a delivery vehicle in previous studies because of its small size and vesicle-like structure. ${ }^{[23-26]}$ In this study, apart from the delivery-related aspect, we rather focused on the natural characteristics of CNVs that support carrying active cell-specific proteins for inducing cellspecific effects. The presence and relative abundance of plasma membrane in CNVs were confirmed using cells expressing fluorescent proteins in their plasma membrane and cytoplasm (Figure 1d,e). In addition, the treatment of CNVs made from trypsin-digested cells clearly showed that the effect of CNVs was mainly induced by the proteins on the plasma membrane (Figure 2d). Taking these two results together, we can reasonably speculate that the effector proteins of CNVs might be carried on their membrane rather than lumen.

On contact with living cells, these active proteins on the $\mathrm{CNV}$ surface give the same contact-dependent stimuli that would be given by their originating cell (Figure 2a). Studies of EVs or exosomes have reported similar concepts. ${ }^{[27]}$ Some of the previous CNV studies highlighted that CNVs and EVs share many characteristics. ${ }^{[23,25]}$ However, according to our analyses, CNVs and EVs share only a few physical characteristics, such as size and structure, but their protein compositions are different: CNVs are more similar to cells than are EVs (Figure 1f). Considering that the CNVs were produced by randomly breaking cells, but the EVs were produced by a complicated biological process, this difference in characteristics is to be expected. Moreover, treatment using EVs isolated from $\mathrm{mEF}$ did not induce the same effects as treatment with CNVs and living feeder cells (Figure S3, Supporting Information). Therefore, we conclude that CNVs are more likely to be cell-like nanovesicles that carry active signaling proteins than are exosomes and EVlike natural messengers. To understand further difference and similarity between CNVs and EVs, more studies are necessary in future.

In typical cell-cell interactions, cells exchange various stimuli through physical contacts, by secreted soluble factors, or both. If a stimulus from a cell-cell interaction is entirely mediated by physical contact between cells, the use of CNVs may replace the function of one cell, and may improve the efficiency of the interaction (Figures $4 \mathrm{a}$ and $5 \mathrm{a}$ ), possibly because cell-CNV interactions have larger interaction surfaces and higher possibilities of interaction than do cell-cell interactions (Figure 2a). When secreted soluble factors are crucial for cell-cell interactions, the use of CNVs, instead of cells, will compromise the efficiency of interactions, or will not induce any interactions at all because CNVs cannot produce or secrete soluble factors. According to our results, $\mathrm{CNV}^{\mathrm{OP} 9}$ and $\mathrm{CNV}^{\mathrm{PA} 6}$ treatments successfully induced ESCs to differentiate into hematopoietic and neuronal cells without any additives; thus, in these cells, the effects of physical contact are dominant. In contrast, $\mathrm{CNV}^{\mathrm{mEF}}$ treatment only reproduced the effect of $\mathrm{mEF}$ feeder layers in the presence of LIF. This result suggests that some of the heterotypic cell-cell interaction between $\mathrm{mEF}$ and ESC is mediated 
a)

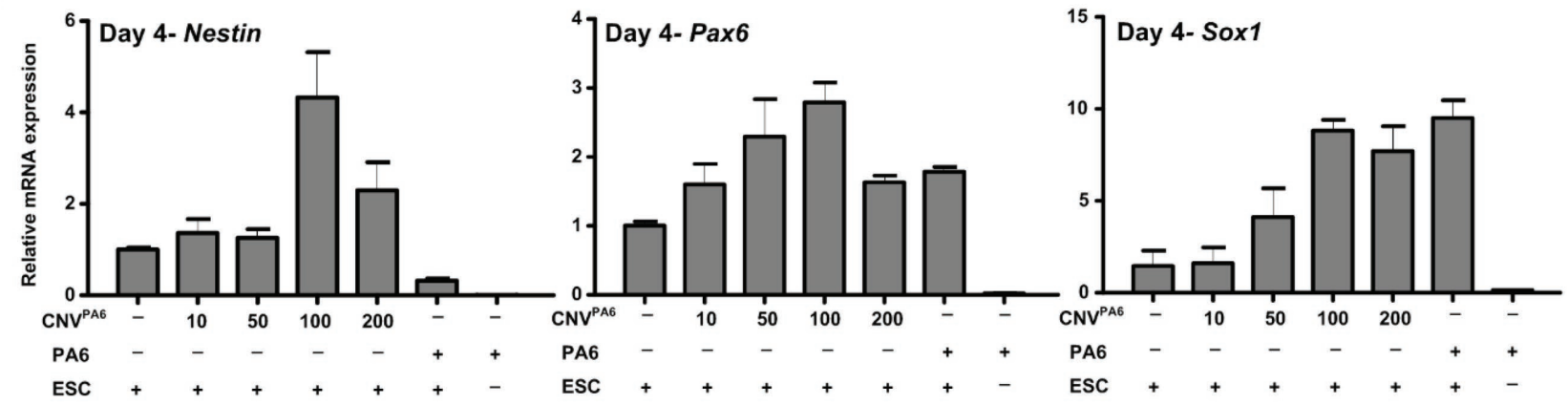

b)

Non-treated

\section{$100 \mu \mathrm{g} \mathrm{CNVPA}$}
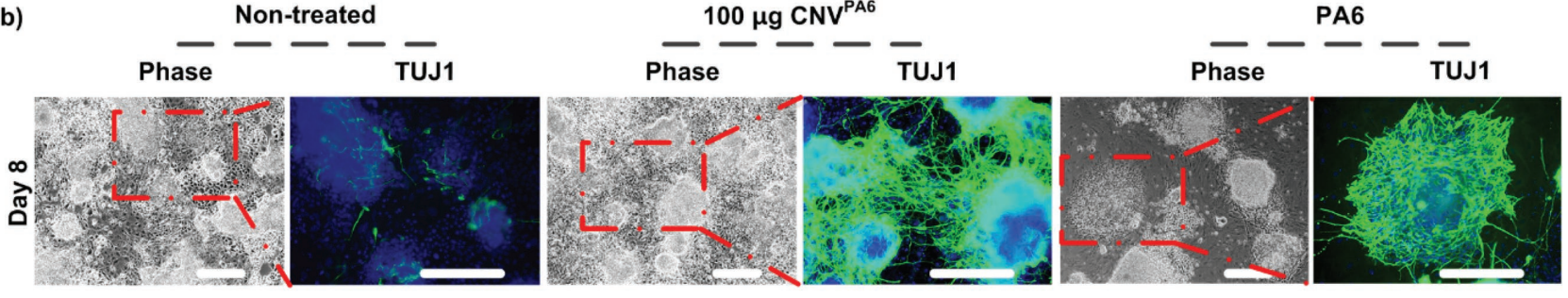

c)
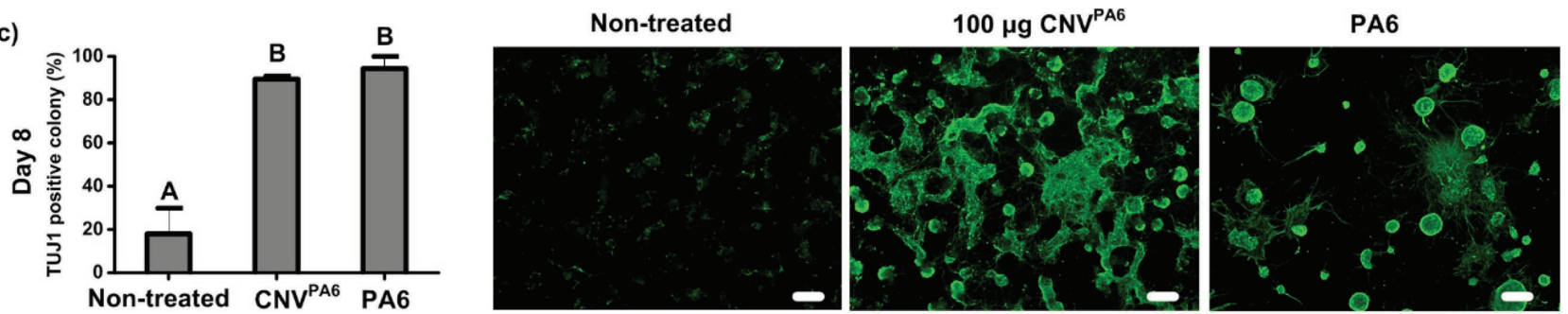

Figure 5. Neuronal differentiation of ESCs after treatment using CNVPA6. a) RT-qPCR analysis of ectodermal genes after PA6 CNV treatment. After two CNV ${ }^{\text {PA6 }}$ treatments, relative gene expressions of three ectodermal genes Nestin, Pax6, and Sox 1 were measured using RT-qPCR. Treatment using $100 \mu \mathrm{g} \mathrm{mL} \mathrm{m}^{-1} \mathrm{CNV}^{\mathrm{PA} 6}$ was optimal for ectodermal differentiation. $n=3$, bars: + S.D. b) Morphologies of ESCs cultured for $8 \mathrm{~d}$ with/without CNVPA6. On the 8th day of culture, ESCs cultured without CNVPA6 showed heterogeneous morphologies of mixed three germ layer cells, whereas ESCs cultured with CNVPA6 or PA6 feeder layers showed homogeneous spindle-like morphologies. Scale bars: $200 \mu \mathrm{m}$. Labelling these cells with neuron-specific TUJ1 antibodies emphasized their morphological differences: ESCs cultured without CNVPA6 showed very few TUJ1-positive cells, but ESCs cultured with $\mathrm{CNV}^{\mathrm{PA} 6}$ and PA6 feeder layers showed increased numbers of TUJ1-positive cells. Scale bars: $200 \mu \mathrm{m}$. c) The percentage of TUJ1-positive colonies on the 8th day of the cultures, as counted in low-magnification images. Bars labeled with the same letter are not significantly different $(p<0.01) ; n=3$, error bars: +S.D.

by soluble factors, which might not be successfully induced by the CNVs. These explanations actually correspond well with previous reports that demonstrated that mEF affects ESCs both by physical contact and by soluble factors, while PA6 mainly affects ESCs by physical contact and matrix deposition. ${ }^{[10,12]}$

Numerous soluble factors in cell-cell interactions have been discovered and produced as recombinant proteins. For example, recombinant LIF and basic fibroblast growth factor have been utilized to maintain ESC self-renewal, and various interleukins and colony-stimulating factors have been used to induce hematopoietic differentiation of ESCs. ${ }^{[13,14,28]}$ In contrast, as of yet, no other methods have been identified that can induce cell-specific contact-dependent stimuli, except coculture methods. Some studies have attempted to solve this problem by using different combinations of ECMs because some part of contact-dependent stimuli are actually induced by ECMs. ${ }^{[16-18]}$ However, these methods did not provide cell-specific contactdependent stimuli mediated by membrane-associated proteins of cells, and therefore they have succeeded only in limited applications. ${ }^{[19]}$ Furthermore, since the membrane-associated molecules of feeder cells that might induce ESC-regulating stimuli have not yet been clearly identified, approaches using defined or purified proteins would not be suitable for providing such cell-specific contact-dependent stimuli. ${ }^{19]}$

The CNV method that we demonstrated in this study constitutes an effective and novel approach. CNVs can be produced from all types of cells. Therefore, if hard to culture cells such as primary cells were used to produce CNVs, we could effectively transmit their stimulus without culturing them. Produced CNVs can be also stored at $-80{ }^{\circ} \mathrm{C}$ for at least three months without noticeable loss of their biological activity. In addition, CNVs can freely diffuse like soluble factors or EVs, and the amount of CNVs can be precisely quantified in terms of particle counts or protein amounts. For these reasons, CNVs can be used simply by mixing them in culture media, which is the same manner in which other soluble factors are used. 
Because, unlike feeder layers, CNVs are freely diffusible, they might be also mixed in suspension cultures such as blood cell cultures and bioreactors to provide contact dependent stimulus. By adjusting the concentration of CNVs in culture media, the amount of stimuli given to treated cells can also be manipulated, which is almost impossible in the case of conventional coculture method. Due to this scalability, we were able to optimize CNV concentrations in terms of protein amounts in all cases. Although we tested the ability of CNV methods only with mouse ESCs, it can be expected to be used in various other applications. In this study, we successfully reproduced three different cocultures consist of different sets of cells and having different mechanism of actions. Based on the results, we can assume that the CNV method has potential to reproduce other pre-established cocultures including human ESCs. ${ }^{[29-31]}$

\section{Conclusion}

The optimized CNV treatments successfully induced contactdependent stimuli in the absence of living cells, and they led to changes in ESCs to the directions that we anticipated. In addition, CNVs can be utilized in combination with other culture additives, such as cytokines and ECMs. In such combination, they can compensate for the weaknesses of each other's methods and produce substantially better results.

\section{Experimental Section}

Cell Culture: C57BL/ 6 mouse ESC, ES-D3 mouse ESC, and OP9 cell lines were purchased from ATCC. MC3T3-PA6 (PA6) cells were purchased from RIKEN Bioresource Center. mEFs were isolated from $13.5 \mathrm{~d}$ old C57BL/ 6 mouse embryos. ${ }^{[32]}$ The procedures using mice were approved by the Institutional Animal Care and Use Committee at POSTECH, Pohang, Republic of Korea (approval number: 2013-01-0016). C57BL/6 and ES-D3 mouse ESCs were maintained on mEF feeder cells in ES media: knock-out Dulbecco Modified Eagle Medium (KO DMEM, Gibco) supplemented with $15 \%$ knock-out serum replacement (KOSR, Gibco), 1\% penicillinstreptomycin (PS, Gibco), $4 \times 10^{-3} \mathrm{M}$ L-glutamine (Sigma-Aldrich), $0.1 \times 10^{-3} \mathrm{M} 2$ 2-mercaptoethanol (2-ME, Sigma-Aldrich), and $1000 \mathrm{U} \mathrm{mL} \mathrm{L}^{-1}$ LIF (ORF Genetics). mEF cells were maintained in DMEM (Gibco) supplemented with 10\% Fetal Bovine Serum (FBS, Hyclone) and 1\% PS. OP9 cells were maintained in Minimum Essential Media (MEM- $\alpha$, Gibco) supplemented with $20 \%$ FBS and $1 \%$ PS. PA6 cells were maintained in MEM- $\alpha$ supplemented with $10 \%$ FBS and $1 \%$ Penicillin-Streptomycin. All cells were cultured on a tissue culture dish coated with $0.2 \%$ gelatin. The $\mathrm{mEF}$ and OP9 feeder layers were prepared by treating confluent monolayers of cells with $10 \mu \mathrm{g} \mathrm{mL}-1$ mitomycin $\mathrm{C}$ for $2 \mathrm{~h}$ at $37^{\circ} \mathrm{C}$. PA6 feeder layers were used without additional growth-arrest procedure.

CNV Production: To preserve membrane proteins, $\mathrm{mEF}$, OP9, and PA6 cells were harvested using nonenzymatic $2 \times 10^{-3} \mathrm{M}$ Ethylenediaminetetraacetic acid (EDTA)/phosphate buffered saline (PBS). The cells were centrifuged at $500 \times g$ for $10 \mathrm{~min}$ at RT, and suspended $\left(2 \times 10^{7}\right.$ cells $\left.\mathrm{mL}^{-1}\right)$ in PBS. To produce Dil-labeled CNVs, cells were stained at this step with vibrant Dil dye according to the manufacturer's instructions. To produce trypsinized CNVs, cells were tripsinized for $30 \mathrm{~min}$ at $37^{\circ} \mathrm{C}$, and then extruded five times each through 10 and $5 \mu \mathrm{m}$ pore polycarbonate track-etched membrane (Whatman) that were installed in a mini-extruder (Avanti). The extruded cells were precleaned by centrifugation at $500 \times \mathrm{g}$ for $10 \mathrm{~min}$ to remove unbroken cells. The supernatants were then applied to iodixanol (Opti-perp, AXIS-SHIELD) density-gradient ultracentrifuge. Briefly, $1 \mathrm{~mL}$ of $10 \%$ iodixanol solution was layered on top of $1 \mathrm{~mL} 30 \%$ iodixanol solution in a $5 \mathrm{~mL}$ ultracentrifuge tube for SW55Ti rotor (Beckman Coulter). The precleaned supernatants were then layered on top of the preformed density gradient and ultracentrifuge with SW55Ti rotor at $100000 \times \mathrm{g}$ for $2 \mathrm{~h}$ at $4{ }^{\circ} \mathrm{C}$. The CNVs were obtained from the layer between the $10 \%$ and $30 \%$ iodixanol. The amount of CNVs was quantified using Bradford protein assay (Sigma-Aldrich). Produced CNVs were mixed with fullgrowth media and cultured for $3 \mathrm{~d}$ in an empty 6 well plate at a $5 \% \mathrm{CO}_{2}$ incubator. After $3 \mathrm{~d}$ of culture, the plate was visually inspected under phase contrast microscope and double checked with CCK-8 cell viability test kit. Aliquots of CNVs could be stored for at least three months at $-80^{\circ} \mathrm{C}$ for further use.

TEM: TEM (JEOL) was used to visualize the morphologies of CNVs. CNVs were dried on a formvar carbon film (FCF300-cu, Electron Microscopy Science), and then negatively stained using $7 \mu \mathrm{L}$ of $2 \%$ uranyl acetate for $10 \mathrm{~s}$. Samples were dried for at least $30 \mathrm{~min}$, and then imaged at $60 \mathrm{kV}$ acceleration voltage.

NTA: The numbers and sizes of CNVs were measured using NTA (Nanosight LM10, Malvern Instruments). The CNVs were diluted in PBS until appropriate numbers of CNVs were detected, and then recorded five times.

Fluorescence Intensity Measurement: Fluorescence intensity of HEK293 cells that expressed palmitoylated mCherry and cytoplasmic GFP, and CNVs made from this cell line were measured using a multiplate reader (DTX880, Beckman). Filter sets for GFP and mCherry measurement were 488/535 and 535/595 (ex/em), respectively.

Confocal Microscopy: For confocal microscopy of cells treated with CNVs, cells were labeled with a CFSE cell tracer (Molecular Probes), and CNVs were labeled with Dil lipophilic dye (Thermo Fisher Scientific). After treatment, cells were fixed with $4 \%$ paraformaldehyde and counter stained with Hoechst (Sigma-Aldrich). Cross-section images were obtained using a confocal microscope (Olympus).

Coomassie Blue Staining: EV, CNV, cell were lysed using $1 \times$ Radioimmunoprecipitation assay (RIPA) buffer, and then quantified using a bicinchoninic acid assay kit (BCA, Thermo Fisher Scientific). The isolated proteins were then denatured by boiling at $100{ }^{\circ} \mathrm{C}$ for $10 \mathrm{~min}$, then separated on SDS-PAGE using electrophoresis at $100 \mathrm{~V}$ for $2 \mathrm{~h}$. The gel was then transferred in fixing solution $(50 \%$ methanol and $10 \%$ glacial acetic acid) for $10 \mathrm{~min}$. After washed in water 3 times with distilled water, the gel was soaked in staining solution $(0.1 \%$ coomassie brilliant blue, $50 \%$ methanol, and $10 \%$ glacial acetic acid) for $30 \mathrm{~min}$ with gentle agitation. Finally, the gel was soaked in destaining solution (40\% methanol and 10\% glacial acetic acid) with gentle agitation and then transferred to fresh destaining solution. The destaining process was repeated until background of the gel was destained.

Western Blot: For western blot to detect phosphorylation of MAPK by short-term CNV treatment, mEFs were serum-starved for $12 \mathrm{~h}$, and then treated with $C N V^{E S C}$ for 30 min. For western blot to detect phosphorylation of AKT by long-term CNV treatment, ESCs were treated with CNVmEF five times for $10 \mathrm{~d}$. The cells were then lysed using $1 \times$ RIPA buffer. Proteins were quantified using a BCA. The isolated proteins were denatured by boiling at $100{ }^{\circ} \mathrm{C}$ for $10 \mathrm{~min}$, and then separated on SDS-PAGE using electrophoresis at $100 \mathrm{~V}$ for $2 \mathrm{~h}$. Separated protein was then transferred to a polyvinylidene fluoride membrane at $390 \mathrm{~mA}$ for $2 \mathrm{~h}$. The proteins were incubated with $5 \%$ nonfat milk in $0.05 \%$ tween- 20 tris-buffered saline (TBST) for $1 \mathrm{~h}$ at RT. AKT and MAPK antibodies were diluted 1:1000 in $5 \%$ nonfat milk in $0.05 \%$ TBST; p-AKT and p-MAPK antibodies were diluted 1:500 in 1\% nonfat milk in $0.05 \%$ TBST. Diluted antibodies were incubated overnight at $4{ }^{\circ} \mathrm{C}$, and then washed three times with $0.05 \%$ TBST. HRP conjugated secondary antibodies (Santa Cruz) were diluted 1:5000 in 5\% nonfat milk in $0.05 \%$ TBST, and then incubated for $1 \mathrm{~h}$ at RT. The immune-reactive bands were detected by applying chemiluminescent substrate (Amersham Pharmacia Biotech) to the membrane. Antibodies used in this research are listed in Table S1 (Supporting Information).

CNV ${ }^{m E F}$ Treatment Conditions: First, ESCs were treated with CNVmEF in the absence of LIF. ESCs were treated with CNVmEF every $2 d$ with medium change for $3 \mathrm{~d}$. On the $3 \mathrm{rd}$ day, cells were observed under phase-contrast microscope IX71 (Olympus) and applied to OCT3/4 flow cytometric analysis. Second, ESCs were treated with CNVmEF 
in the presence of LIF every $2 \mathrm{~d}$ for $24 \mathrm{~d}$. On the 12th day, cells were observed under phase contrast microscope and applied to OCT3/4 flow cytometric analysis. On the 24th day, cells were stained for ALP, $\mathrm{OCT} 3 / 4$, and SOX2, and observed under a fluorescence microscope IX71 (Olympus). The cells from the 24th day were also applied to EB formation and differentiation.

CNVOP9 Treatment Conditions: ESCs were treated with CNVOP9 in hematopoietic differentiation medium (MEM- $\alpha$ supplemented with $20 \%$ FBS, $1 \%$ PS, $0.1 \times 10^{-3}$ M 2-ME and $1 \%$ nonessential amino acids). Feeder control groups were cultured as previously described. ${ }^{[33]}$ CNVOP9 were applied every $3 \mathrm{~d}$ for $17 \mathrm{~d}$. On the 6th day, cells were observed under phase-contrast microscope and applied to RT-qPCR for Flk 1 and $\mathrm{Scl}$ for dose optimization. On the 17th day, cells were observed under phase-contrast microscope, and harvested for flow cytometric analyses of c-KIT, SCA1, CD45, MAC1, GR1, TER119, B220, and CD3 hematopoietic cell markers (PCR primers and antibodies are listed in Tables S1 and S2 in the Supporting Information).

CNV ${ }^{P A 6}$ Treatment Conditions: ESCs were treated with CNV ${ }^{\text {PA6 }}$ in neural differentiation medium (KO DMEM supplemented with 15\% KOSR, 1\% $\mathrm{PS}, 4 \times 10^{-3} \mathrm{M}$ L-glutamine, and $\left.0.1 \times 10^{-3} \mathrm{M} 2-\mathrm{ME}\right)$. Feeder control groups were cultured as previously described. ${ }^{[12]} \mathrm{CNV}^{\mathrm{PA} 6}$ were applied every $2 \mathrm{~d}$ for $8 \mathrm{~d}$. On the 4th day, cells were observed under phase-contrast microscope and applied to RT-qPCR for Nestin, PaxG, and Sox 1 for dose optimization. On the 8th day, cells were observed under phase-contrast microscope, and stained for TUJ1 neuron marker for visualization (PCR primers and antibodies are listed in the Supplementary data). Fluorescence images were taken, and then Image) software $(\mathrm{NIH})$ was used to calculate the percentage of TUI1 positive colonies.

$R T-q P C R$ : RNAs were isolated using Isol-RNA reagent (5 PRIME). The concentrations of isolated RNAs were quantified using a SimpliNano (GE). A reverse transcription kit (Promega) was used to generate CDNAs from the RNAs. Quantitative PCR was conducted using the MyiQ Real-time PCR Detection System (Bio-Rad) and iQ SYBR Green Supermix (Bio-Rad). Expression levels of all genes were normalized to the expression of $\beta$-actin. Primers used in RT-qPCR are listed in Table S2 (Supporting Information).

Flow Cytometry: Immunostained samples were analyzed using an LSR Fortessa flow cytometer (BD Biosciences). All data were analyzed and plotted using Flowing software (http://www.uskonaskel.fi/ flowingsoftware/).

Immunostaining: The cell cultures were fixed with $4 \%$ paraformaldehyde (Sigma-Aldrich) for $10 \mathrm{~min}$ at RT, and then washed three times with $0.05 \%$ Tween 20 in PBS (0.05\% PBST). Then, $0.2 \%$ Triton X-100 (Sigma-Aldrich) in PBS was added to each sample for $10 \mathrm{~min}$ at RT to permeabilize the cells, which were then washed three times with PBST. Samples were incubated in blocking solution $(5 \%$ FBS in PBS, $5 \%$ horse serum in PBS, or 3\% BSA in PBS) at RT for $1 \mathrm{~h}$. The samples were incubated with primary antibody solutions overnight at $4{ }^{\circ} \mathrm{C}$, and then washed three times with PBST. Secondary antibodies were added to the samples and incubated at RT for $1 \mathrm{~h}$, and then the samples were washed three times with PBST. The samples were counterstained with Hoechst (Sigma-Aldrich) at RT for $10 \mathrm{~min}$, and then washed three times with PBST. Images were observed using a fluorescence microscope IX71 (Olympus). Antibodies used in this research are listed in Table S1 (Supporting Information).

Statistical Analysis: All statistical analyses were performed using $\mathrm{R}$ software (https://www.r-project.org/). The normality of the results was tested using the Shapiro-Wilk normality test. The homogeneity of variances of the results was tested using Levene's test. The statistical significance of the results was analyzed using one-way between-groups analysis of variance, and significance between pairs was analyzed using Tukey's HSD post hoc tests.

\section{Supporting Information}

Supporting Information is available from the Wiley Online Library or from the author.

\section{Acknowledgements}

J.K. and C.H. contributed equally to this work. This research was supported by a grant of the Korea Health Technology R\&D Project through the Korea Health Industry Development Institute (KHIDI), funded by the Ministry of Health and Welfare, Republic of Korea (grant no. HI16C2221) and partially supported by National Research Foundation of Korea (NRF) grant funded by the Korean Government (MSIP) (No. 2011-0028845).

\section{Conflict of Interest}

The authors declare no conflict of interest.

\section{Keywords}

cell-cell interaction, cell-engineered nanovesicles, culture additives, membrane vesicles, stem cell regulation

Received: March 24, 2017

Revised: May 11, 2017

Published online: June 23, 2017

[1] D. L. Chao, L. Ma, K. Shen, Nat. Rev. Neurosci. 2009, 10, 262.

[2] S. K. Biswas, A. Mantovani, Nat. Immunol. 2010, 11, 889.

[3] N. Perrimon, C. Pitsouli, B.-Z. Shilo, Cold Spring Harbor Perspect. Biol. 2012, 4, a005975.

[4] F. Yue, S. Shirasawa, H. Ichikawa, S. Yoshie, A. Mogi, S. Masuda, M. Nagai, T. Yokohama, T. Daihachiro, K. Sasaki, in Regenerative Medicine and Tissue Engineering, InTech, 2013, Ch 5, pp. 117-139.

[5] S. Roy, T. B. Kornberg, BioEssays 2015, 37, 25.

[6] F. Fagotto, B. M. Gumbiner, Dev. Biol. 1996, 180, 445.

[7] G.-I. Im, Tissue Eng., Part B 2014, 20, 545.

[8] Q. Chen, J. Wu, Q. Zhuang, X. Lin, J. Zhang, J.-M. Lin, Sci. Rep. 2013, 3, 2433

[9] D. G. Halme, D. A. Kessler, N. Engl. J. Med. 2006, 355, 1730.

[10] G. Tremml, M. Singer, R. Malavarca, Current Protocols in Stem Cell Biology, John Wiley \& Sons, Inc., Hoboken, NJ, USA, 2008, pp. 1-19.

[11] T. Nakano, H. Kodama, T. Honjo, Science 1994, 265, 1098.

[12] H. Kawasaki, K. Mizuseki, S. Nishikawa, S. Kaneko, Y. Kuwana, S. Nakanishi, S.-I. Nishikawa, Y. Sasai, Neuron 2000, 28, 31.

[13] Q.-L. Ying, J. Wray, J. Nichols, L. Batlle-Morera, B. Doble, J. Woodgett, P. Cohen, A. Smith, Nature 2008, 453, 519.

[14] H. Niwa, T. Burdon, I. Chambers, A. Smith, Genes Dev. 1998, 12, 2048.

[15] Y. Xu, Y. Shi, S. Ding, Nature 2008, 453, 338.

[16] F. M. Watt, W. T. S. Huck, Nat. Rev. Mol. Cell Biol. 2013, 14, 467

[17] C. J. Flaim, S. Chien, S. N. Bhatia, Nat. Methods 2005, 2, 119.

[18] F. Guilak, D. M. Cohen, B. T. Estes, J. M. Gimble, W. Liedtke, C. S. Chen, Cell Stem Cell 2009, 5, 17.

[19] S.-I. Nishikawa, L. M. Jakt, T. Era, Nat. Rev. Mol. Cell Biol. 2007, 8, 502.

[20] X.-S. Yue, M. Fujishiro, C. Nishioka, T. Arai, E. Takahashi, J.-S. Gong, T. Akaike, Y. Ito, PLoS One 2012, 7, e32707.

[21] Y. Zhou, H. Mao, B. Joddar, N. Umeki, Y. Sako, K.-I. Wada, C. Nishioka, E. Takahashi, Y. Wang, Y. Ito, Sci. Rep. 2015, 5, 11386

[22] N. Berger, A. Sachse, J. Bender, R. Schubert, M. Brandl, Int. J. Pharm. 2001, 223, 55.

[23] S. C. Jang, O. Y. Kim, C. M. Yoon, D.-S. Choi, T.-Y. Roh, J. Park, J. Nilsson, J. Lötvall, Y.-K. Kim, Y. S. Gho, ACS Nano 2013, 7, 7698. 
[24] A. S. Cheung, S. T. Koshy, A. G. Stafford, M. M. C. Bastings, D. J. Mooney, Small 2016, 12, 2321.

[25] Y. Jin, J. S. Lee, S. Min, H.-J. Park, T. J. Kang, S.-W. Cho, Adv. Funct. Mater. 2016, 26, 5804.

[26] O. Y. Kim, S. J. Choi, S. C. Jang, K. S. Park, S. R. Kim, J. P. Choi, J. H. Lim, S. W. Lee, J. Park, D. Di Vizio, J. Lötvall, Y. K. Kim, Y. S. Gho, Nano Lett. 2015, 15, 266.

[27] M. Yáñez-Mó, P. R.-M. Siljander, Z. Andreu, A. Bedina Zavec, F. E. Borràs, E. I. Buzas, K. Buzas, E. Casal, F. Cappello, J. Carvalho, E. Colás, A. Cordeiro-da Silva, S. Fais, J. M. FalconPerez, I. M. Ghobrial, B. Giebel, M. Gimona, M. Graner, I. Gursel, M. Gursel, N. H. H. Heegaard, A. Hendrix, P. Kierulf, K. Kokubun, M. Kosanovic, V. Kralj-Iglic, E.-M. Krämer-Albers, S. Laitinen, C. Lässer, T. Lener, E. Ligeti, A. Linē, G. Lipps, A. Llorente, J. Lötvall, M. Manček-Keber, A. Marcilla, M. Mittelbrunn, I. Nazarenko,
E. N. M. Nolte-'t Hoen, T. A. Nyman, L. O'Driscoll, M. Olivan, C. Oliveira, É. Pállinger, H. A. del Portillo, J. Reventós, M. Rigau, E. Rohde, M. Sammar, F. Sánchez-Madrid, N. Santarém, K. Schallmoser, M. Stampe Ostenfeld, W. Stoorvogel, R. Stukelj, S. G. Van der Grein, M. H. Vasconcelos, M. H. M. Wauben, O. De Wever, J. Extracell. Vesicles 2015, 4, 27066.

[28] K. Chadwick, Blood 2003, 102, 906.

[29] S. L. McElroy, R. A. R. Pera, Cold Spring Harbor Protoc. 2008, 3, pdb-prot5041.

[30] M. A. Vodyanik, J. A. Bork, J. A. Thomson, I. I. Slukvin, Blood 2005, 105, 617.

[31] X. Zeng, J. Cai, J. Chen, Y. Luo, Z. You, E. Fotter, Y. Wang, B. Harvey, T. Miura, C. Backman, Stem Cells 2004, 22, 925

[32] K. Takahashi, S. Yamanaka, Cell 2006, 126, 663.

[33] P.-K. Chen, G.-L. Lin, H.-H. Chang, D.-S. Sun, Exp. Cell Res. 2015, 339, 44. 